\title{
Met-myoglobin formation, accumulation, degradation, and myoglobin oxygenation monitoring based on multiwavelength attenuance measurement in porcine meat
}

\author{
Thien Nguyen \\ Kien Nguyen Phan \\ Jee-Bum Lee \\ Jae Gwan Kim
}

Thien Nguyen, Kien Nguyen Phan, Jee-Bum Lee, Jae Gwan Kim, "Met-myoglobin formation, accumulation, degradation, and myoglobin oxygenation monitoring based on multiwavelength attenuance

SPIE measurement in porcine meat," J. Biomed. Opt. 21(5), 057002 (2016), doi: 10.1117/1. 


\title{
Met-myoglobin formation, accumulation, degradation, and myoglobin oxygenation monitoring based on multiwavelength attenuance measurement in porcine meat
}

\author{
Thien Nguyen, ${ }^{a}$ Kien Nguyen Phan, ${ }^{b}$ Jee-Bum Lee, ${ }^{c}$ and Jae Gwan Kim ${ }^{a, *}$ \\ aGwangju Institute of Science and Technology, Department of Medical System Engineering, 123 Cheomdangwagi-ro, Buk-gu, Gwangju 61005, \\ Republic of Korea \\ ${ }^{\mathrm{b} H a n o i}$ University of Science and Technology, Department of Electronics Technology and Biomedical Engineering, 1 Dai Co Viet Road, \\ Hanoi 10000, Vietnam \\ ${ }^{\circ}$ Chonnam National University Medical School, Department of Dermatology, 42 Jebong-ro, Dong-gu, Gwangju 61469, Republic of Korea
}

\begin{abstract}
We propose a simple, rapid, and nondestructive method to investigate formation, accumulation, and degradation of met-myoglobin (met-Mb) and myoglobin oxygenation from the interior of porcine meat. For the experiment, color photos and attenuance spectra of porcine meat (well-bled muscle, fat, and mixed) were collected daily to perform colorimetric analysis and to obtain the differences of attenuance between 578 and $567 \mathrm{~nm}$ (A578-A567) and between 615 and $630 \mathrm{~nm}$ (A630-A615), respectively. Oxy-, deoxy-, and met-myoglobin concentration changes over storage time were also calculated using Beer-Lamberts' law with reflectance intensities at 557,582 , and $630 \mathrm{~nm}$. The change of A578-A567 was well matched with the change of myoglobin oxygenation, and the change of A630-A615 corresponded well with the formation and degradation of met-Mb. In addition, attenuation differences, A578-A567 and A630-A615, were able to show the formation of met-Mb earlier than colorimetric analysis. Therefore, the attenuance differences between wavelengths can be indicators for estimating myoglobin oxygenation and met-Mb formation, accumulation, and degradation, which enable us to design a simple device to monitor myoglobin activities in porcine meat. ๑ 2016 Society of Photo-Optical Instrumentation Engineers (SPIE) [DOI: 10.1117/1.JBO.21.5.057002]
\end{abstract}

Keywords: oxy-myoglobin; deoxy-myoglobin; met-myoglobin; porcine meat; meat discoloration.

Paper 150814RR received Dec. 6, 2015; accepted for publication Apr. 13, 2016; published online May 6, 2016.

\section{Introduction}

Meat discoloration is a direct cause of consumer's rejection because purchasers usually use color change as an indicator of spoilage. ${ }^{1,2}$ Previous studies found that the meat color transformation mainly resulted from different proportions of myoglobin species., ${ }^{3,4}$ Myoglobin is the most dominant pigment in muscle, which normally exists in cut meat as oxy-myoglobin (oxy-Mb), deoxy-myoglobin (deoxy-Mb), and met-myoglobin (met-Mb). Oxy-Mb has a bright red color, corresponding to fresh meat, deoxy-Mb has a purplish red color, and met-Mb has a brownish color. ${ }^{2,5,6}$ An increase in surface met-Mb percentage turns meat to a brown color and makes it become unsaleable. ${ }^{3}$ Devine and Dikeman ${ }^{7}$ reported that the consumers start to discriminate meat when $20 \%$ met-Mb is detected. An offset against meat discoloration is discount in price, which brings about a billion-dollar loss each year. ${ }^{1}$

Figure 1 models the change of myoglobin species' distribution in the cut meat over storage time conceptually. When meat is first cut, the meat surface is quickly covered by a thin layer of oxy-Mb due to the oxygenation process, which converts deoxy$\mathrm{Mb}$ to oxy-Mb. The inner layer is occupied by deoxy-Mb. The oxygenation process starts at the meat surface, eventually spreads inward, and continues for several days, ${ }^{7}$ resulting in

*Address all correspondence to: Jae Gwan Kim, E-mail: jaekim@ gist.ac.kr a thicker oxy-Mb layer. In addition to the oxygenation process, the oxidation process, which degrades oxy- and deoxy-Mb to met-Mb, ${ }^{8}$ also occurs in the cut meat, but it first takes place in the interior meat layer and gradually spreads to the meat surface. The oxygenation and oxidation processes divide cut meat into three layers. The uppermost layer, where oxygen is available, accumulates oxy-Mb, while deoxy-Mb occupies the innermost layer, and the met-Mb layer is formed in between. ${ }^{9}$ A previous study found that the met-Mb layer lies between 1 and $6 \mathrm{~mm}$ from the cut surface. ${ }^{9}$ Further storage enhances the oxidation process but diminishes the oxygenation process, resulting in the shrinkage of the oxy-Mb layer and the expansion of the met-Mb layer to the cut surface. ${ }^{3}$ The approach of met-Mb to the surface discolors the cuts, and consequently shortens the meat shelf life.

Various approaches were suggested, such as irradiation and antioxidant, to lengthen meat shelf life by the reduction of met-Mb. ${ }^{3,5,10}$ These methods showed a potential to retain meat color, but their efficiency mainly depended on myoglobin redox chemistry, especially, oxygen consumption and met-Mb formation. ${ }^{1,10}$ As mentioned above, met-Mb formation originates from the inner layer and then spreads to the cut surface. Hence, monitoring of interior met-Mb formation and myoglobin oxygenation may help to improve meat color stabilizing techniques.

1083-3668/2016/\$25.00 @ 2016 SPIE 


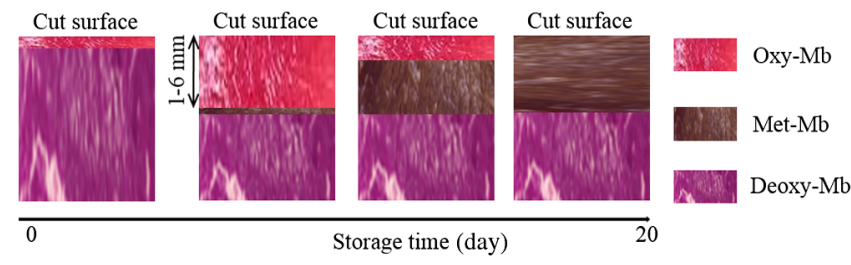

Fig. 1 A conceptual model of myoglobin species' distribution in cut meat over storage time.

Current pigment quantification studies follow two primary directions, light reflection measurement and pigment extraction. Light reflection measurement is rapid and nondestructive, but it only characterizes surface color. The extraction technique can characterize interior pigments, but it is destructive and time consuming. ${ }^{10}$ The disadvantages of previous studies demand a new method, which is simple, rapid, nondestructive, and allows interior measurement. The problem can be solved by a contact spectroscopic approach using a probe with a certain separation between light source and detector. Similar to light reflection measurement, the spectroscopic method is rapid and nondestructive. Additionally, the contact of the probe with a separation between the light source and detector on the surface of the tissue allows us to obtain myoglobin derivative information from the inner layers of tissue approximately half of the source and detector separation. ${ }^{11}$

Previous studies have derived the absorbance spectra of myoglobin derivatives ${ }^{4,12-14}$ and their corresponding peaks. Oxy-Mb has peaks at 418,544 , and $582 \mathrm{~nm}$, deoxy-Mb has peaks at 434 and $557 \mathrm{~nm}$, and met-Mb has peaks at 410,505 , and $634 \mathrm{~nm} .{ }^{4}$ Similarly, Schenkman et al. ${ }^{15}$ found the met-Mb peak at $628 \mathrm{~nm}$, and Krzywicki ${ }^{16}$ claimed the peak at $630 \mathrm{~nm}$. Kerry et al. ${ }^{17}$ affirmed the oxy-Mb peak at 542 and $578 \mathrm{~nm}$. For convenience, we used oxy-Mb peaks at 542 and $578 \mathrm{~nm}$, deoxy-Mb peak at $557 \mathrm{~nm}$, and met-Mb peak at $630 \mathrm{~nm}$. It has been shown that the myoglobin derivatives have extinction coefficients very close to those of hemoglobin $(\mathrm{Hb})$ derivatives. ${ }^{12,18,19}$ Because $\mathrm{Hb}$ derivatives' extinction coefficients are widely available, we use Hb's values ${ }^{20}$ to illustrate myoglobin's absorption spectra [Fig. 2(a)].

The reduced scattering coefficient of the muscle is derived from Rayleigh and Mie scattering [Eq. (1), Fig. 2(a)], with $a=13.0, f_{\text {Ray }}=0.00$ and $b_{\text {Mie }}=0.926$ :

$$
\begin{aligned}
\mu_{\mathrm{s}}^{\prime}(\lambda)= & a\left[f_{\text {Ray }}\left(\frac{\lambda}{500 \mathrm{~nm}}\right)^{-4}\right. \\
& \left.+\left(1-f_{\text {Ray }}\right)\left(\frac{\lambda}{500 \mathrm{~nm}}\right)^{-b_{\text {Mie }}}\right],
\end{aligned}
$$

where $a$ is the reduced scattering coefficient at $500 \mathrm{~nm}, f_{\text {Ray }}$ is the fraction of Rayleigh scattering, and $b_{\text {Mie }}$ is the power of Mie scattering. ${ }^{21}$

As shown in Fig. 2(a), in the 500- to 700-nm range, the absorption is dominant over the scattering; hence, in this range, the attenuation, which is the summation of absorption and scattering, mainly results from the absorption. In addition, the scattering coefficient gradually decreases with a factor of $b_{\text {Mie }}=0.926$, so the addition of the scattering may minimally alter the attenuance, but not the peak behavior compared with the absorbance. As a result, in the 500- to 700-nm range, oxy-, deoxy-, and met-Mb have attenuation spectra very similar to their absorption spectra [Fig. 2(b)].

Formerly, researchers predicted myoglobin species' concentration on the meat's surface using the $K / S 572-K / S 525$ value, ${ }^{19,22,23}$ where $K$ is the absorption coefficient, $S$ is the scattering coefficient, and the $K / S$ value was derived from the Kubelka-Munk equation. ${ }^{24,25}$ Utilization of the $K / S$ value helps account for both absorption and scattering, but it requires the reference values for $100 \%$ of the three meat pigment forms, which are unique for each experimental condition. ${ }^{26}$ Furthermore, these methods could estimate only the surface portion.

Hence, in this study, a spectroscopic-based method was applied to monitor myoglobin oxygenation and the formation, accumulation, and degradation of met-Mb in porcine meat. Both muscle and fat were considered to investigate myoglobin's role. Finally, various depths were taken into account to find an appropriate depth for observation of all myoglobin species' proportion change.

\section{Material and Methods}

\subsection{Meat Sample Preparation}

The experiments were conducted twice, one was in winter, with an average temperature of $-5^{\circ} \mathrm{C}$, and the other was in summer, with an average temperature of $25^{\circ} \mathrm{C}$. Porcine meat was
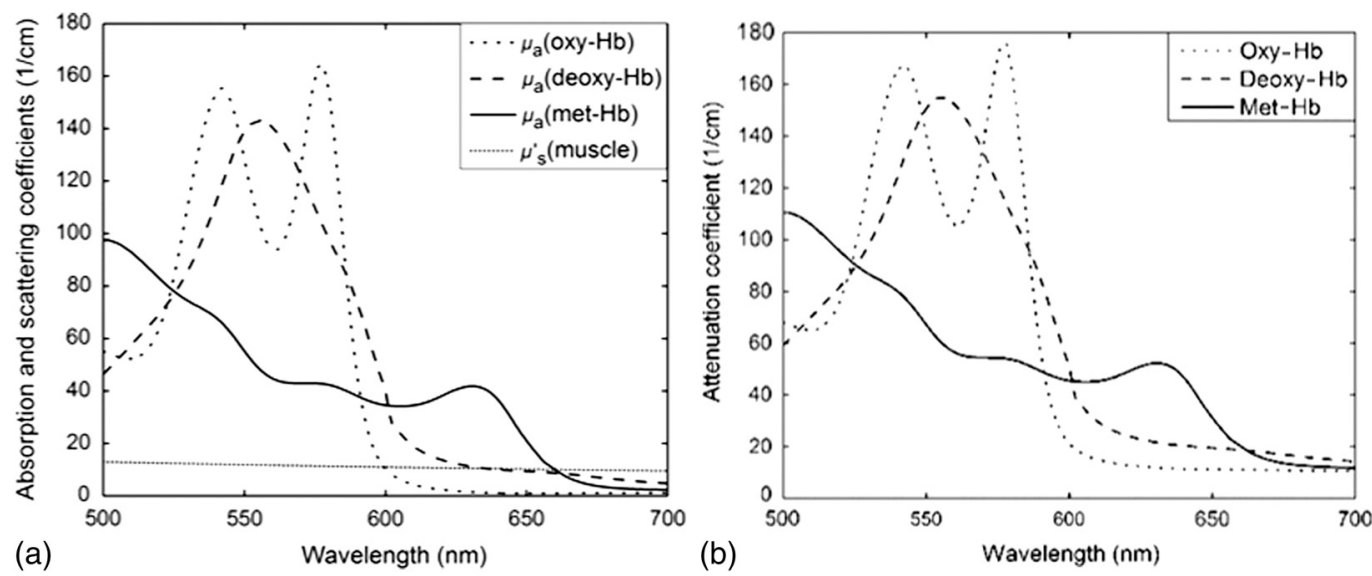

Fig. 2 Tissue optical property: (a) the absorption spectra of oxy-, deoxy-, and met- $\mathrm{Hb}$ and the reduced scattering spectrum of the muscle, and (b) the attenuation (summation of absorption and scattering) spectra of oxy-, deoxy-, and met-Hb. 
purchased from a butcher's shop approximately $1 \mathrm{~h}$ after slaughtering. The meat was collected from different parts of a pig. Three different types of porcine samples, well-bled muscle, mixture of muscle and fat, and fat only samples were taken into account to investigate the effect of various myoglobin concentrations on myoglobin oxygenation and met-Mb formation monitoring. Within $1 \mathrm{~h}$, the meat sections were transported to our experiment room in a cooler filled with ice. Once the meat arrived, samples were prepared. In the first experiment, 52 samples with thicknesses varying from 1 to $3 \mathrm{~cm}$, and weights ranging from 20 to $50 \mathrm{~g}$, were prepared. Among 52 samples, there were 34 well-bled muscle samples, 16 mixture of muscle and fat samples, and 2 fat samples. In the second experiment, there were 52 porcine samples, which were 35 well-bled muscles, 13 mixtures, and 4 fats. Samples' thicknesses varied from 1 to $4 \mathrm{~cm}$, and their weights were from 14 to $46 \mathrm{~g}$. The mixed sample consists of a very thin fat layer in the cut surface and an inner, thick well-bled muscle layer. The fat layer thickness varies from 0.5 to $1 \mathrm{~mm}$, and the well-bled muscle layer thickness ranges from 2 to $3 \mathrm{~cm}$.

\subsection{Meat Storage}

Samples were wrapped separately in polyethylene bags, marked, and stored in a refrigerator at $2^{\circ} \mathrm{C}$, which is similar to the storage conditions at the butcher's shop. Each sample was taken out of the refrigerator three times per day, twice for the measurements of the attenuation spectrum and the samples' weight and once for taking a color photo of the samples. The spectral measurement was conducted in a room with a temperature of $16^{\circ} \mathrm{C}$ for the first experiment during winter and $20^{\circ} \mathrm{C}$ for the second experiment during summer. Measurement time was approximately $1 \mathrm{~min}$ per each sample. The process was repeated every $12 \mathrm{~h}$, up to 10 days postmortem. The total air exposure time for each sample was approximately 3 min per day, which was short enough to neglect the effect of the room conditions on the meat sample.

\subsection{Samples' Photo Collection and Colorimetric Analysis}

All samples' photos were taken once per day at 4 pm to monitor the color change daily. Photo collection was performed in a bright experiment room with a temperature of $18^{\circ} \mathrm{C}$. The samples were placed on a blank A4 paper and a photo was taken using a NX3000 Camera (Samsung, Korea) with white LED illumination. The collected photos were then decomposed to red, green, and blue color components. In order to eliminate differences in light illumination intensity, we calculated the ratios of red, green, and blue colors with respect to the sum of the three components.

\subsection{Spectroscopic Setup}

Broadband light (HL-2000-HP, Ocean Optics, Delray Beach, Florida) was shone onto the samples and the reflectance light was collected by four spectrometers (USB-4000, Ocean Optics). The first spectrometer (S1) collects light between 345- and $1036-\mathrm{nm}$ frequencies with a 500-ms integration time, 0.2-nm resolution, and 3-mm source-detector separation. The other three spectrometers (S2, S3, and S4) measure signals between 470- and 1148-nm frequencies with a 200-ms integration time, $0.2-\mathrm{nm}$ resolution, and the source-detector separations were 5,
10, and $15 \mathrm{~mm}$, respectively. Four different source-detector distances (SD) were set up using five optical fiber probes (Fig. 3) to monitor the attenuation spectra from various interior layers. SD is considered an important factor for the depth of light penetration (DLP) estimation. A previous study suggested that the light is able to attain a DLP equal to half of the SD when the SD is shorter than $40 \mathrm{~mm} .{ }^{11}$ Therefore, based on that limitation, it can be said that the further the SD, the deeper the DLP. Utilization of four different SDs is expected to collect signals from four different DLPs, with a minimum DLP of $1.5 \mathrm{~mm}$ (3 mm SD) and maximum DLP of $7.5 \mathrm{~mm}$ (15 mm SD). Considering the results from these various depths allowed us to investigate the location of the met-Mb layer.

Compared with near-infrared, visible light is highly absorbed by tissue, ${ }^{27}$ so the DLP is limited. In our experiment, only the $3 \mathrm{~mm} \mathrm{SD}$ was expected to be able to collect visible light. Each optical fiber probe (2-mm diameter) consists of seven multimode low $\mathrm{OH}$ - fibers $(400 \mu \mathrm{m})$ bundled with a stainless steel sheath.

\subsection{Spectra Collection}

On the day the meat was obtained from the butcher's shop, the measurement was performed once, at night, referred to as Day0. For the following days, the meat samples were measured every $12 \mathrm{~h}$ (twice per day) in the morning and at night up to Day9. The experiment was conducted in a dark room with all lights off, and all doors and windows were covered by black curtains. From each sample, 19 spectra were collected, a spectrum on Day0 and then 18 others over the next 9 days. The results from both the first and the second experiments consisted of 3952 spectra, which came from 52 samples, 19 time measurements, and 4 different SDs. For each spectrometer, a reference spectrum was collected using an integrating sphere with $99.9 \%$ reflectance (4 in., Edmund Optics).

\subsection{Spectral Analysis}

After collecting all reflectance spectra, attenuance was then calculated as in the following equation:

Attenuance $=-\log \left(\frac{\text { measured intensity }}{\text { reference intensity }}\right)$.

According to the attenuation spectra of oxy-, deoxy-, and met-Mb [Fig. 2(b)], myoglobin oxygenation can be monitored

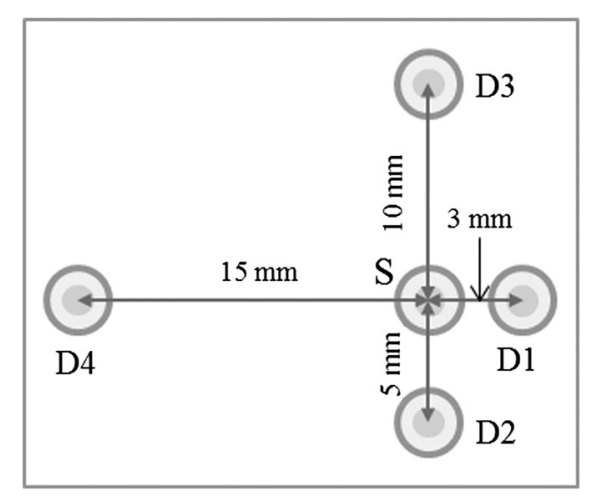

Fig. 3 Probe design. D: detector fiber: connected to spectrometer, and S: light source fiber. 
using light in the range from 525 to $600 \mathrm{~nm}$. This range includes not only 542- and 578-nm oxy-Mb peaks, but also a 557-nm deoxy-peak. When there is $100 \%$ of deoxy-Mb, the attenuation gradually decreases from 557 to $600 \mathrm{~nm}$. While in the presence of oxy-Mb, due to its peak at $578 \mathrm{~nm}$, attenuation increases from 557 to $578 \mathrm{~nm}$, and then decreases. Hence, a predominance of oxy-Mb percentage results in two peaks, while a prevalence of deoxy-Mb proportion yields only one peak. Myoglobin oxygenation increases oxy-Mb portion, but diminishes the deoxy-Mb ratio, resulting in the appearance of oxy-Mb peaks and the vanishing of the deoxy-Mb peak. ${ }^{17}$ As a result, investigation of the attenuation spectrum in the 525- to 600-nm range, consisting of oxy- and deoxy-Mb peaks, can monitor the oxygenation process.

Similarly, examination of the attenuation in the 600- to 665$\mathrm{nm}$ range can help to observe met-Mb formation. In the absence of met-Mb, the attenuation spectrum of myoglobin gradually falls off from 600 to $665 \mathrm{~nm}$, while the addition of met-Mb causes changes in the spectra. Specifically, with the presence of met-Mb, a peak appears at around $630 \mathrm{~nm}$, resulting in higher attenuance by met-Mb from 600 to $630 \mathrm{~nm}$. Consequently, monitoring the attenuation spectrum between 600 and $665 \mathrm{~nm}$ can provide a signature of met-Mb formation and accumulation.

\subsection{Multiwavelength Analysis}

Analysis in Sec. 2.6 pointed out that the 578-nm peak's aprearance can be a sign for myoglobin oxygenation. A simple way to detect the peak is using the difference of the attenuance between two wavelengths, 578 and $567 \mathrm{~nm}$ [A578-A567 difference, Eq. (3), Fig. 3]:

$$
\begin{aligned}
\text { A578-A567 difference }= & \text { attenuance at } 578 \mathrm{~nm} \\
& - \text { attenuance at } 567 \mathrm{~nm} .
\end{aligned}
$$

The reasons to choose 567 and $578 \mathrm{~nm}$ were because they are related to the oxy-Mb peaks, and the met-Mb attenuation coefficient at these two wavelengths is similar (Fig. 2). Without an oxy-Mb 578-nm peak, the decrease of attenuance from 557 to $600 \mathrm{~nm}$ yields a negative A578-A567 difference [Fig. 4(a)], while the presence of the 578-nm peak drives the A578-A567
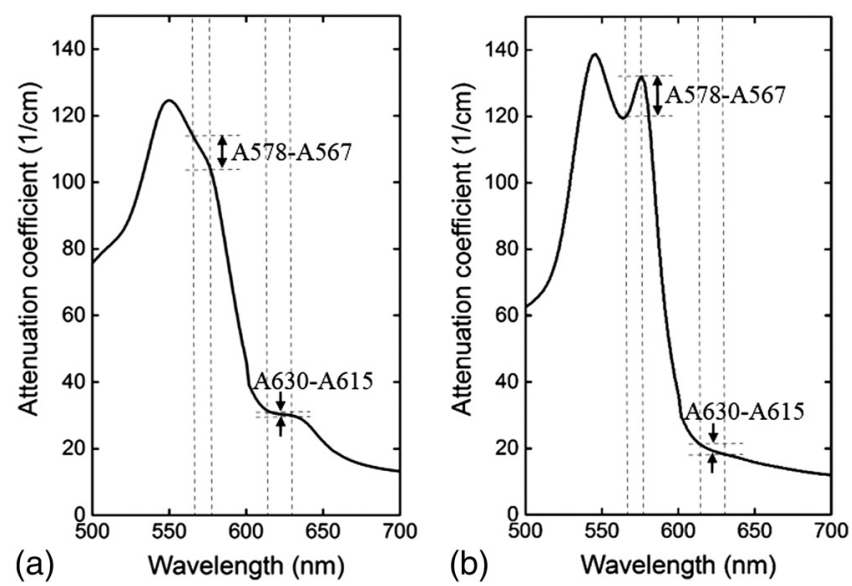

Fig. 4 Demonstration for A578-A567 and A630-A615 calculations with different myoglobin proportions: (a) 10\% oxy-Mb, $60 \%$ deoxy$\mathrm{Mb}$, and $30 \%$ met-Mb, and (b) $45 \%$ oxy-Mb, $50 \%$ deoxy-Mb, and $5 \%$ met-Mb. difference to be positive [Fig. 4(b)]. Similarly, we used the A630-A615 difference [Eq. (4), Fig. 4] to monitor met-Mb formation and accumulation:

$$
\begin{aligned}
\mathrm{A} 630-\mathrm{A} 615 \text { difference }= & \text { attenuance at } 630 \mathrm{~nm} \\
& - \text { attenuance at } 615 \mathrm{~nm} .
\end{aligned}
$$

A previous study employed the attenuation ratios of the peaks of oxy-Mb and deoxy-Mb (A485/A560 and A635/A560) to examine those species' presence. ${ }^{13}$ They claimed that by considering both species' peaks, the advantage of monitoring both the reduction in oxy-Mb and the expansion of met-Mb at once could be obtained. However, oxy-Mb attenuance is approximately three times higher than met-Mb attenuance at their corresponding peaks (Fig. 2), which weakens the summation effect. Alternatively, the relatively close attenuation coefficient at the two wavelengths of 615 and $630 \mathrm{~nm}$ allows for the observation of even the slightest change in met-Mb concentration. In addition, utilization of the difference instead of the ratio can remove the effect of dark noise. The difference maintains its value regardless of either the addition or the subtraction of a dark noise, but the dark noise alters the result if the ratio is used, as in the following equation:

$$
A x-A y=(A x \pm a)-(A y \pm a) ; \quad \frac{A x}{A y} \neq \frac{A x \pm a}{A y \pm a},
$$

where $a$ is the dark noise. Instead of using the attenuance difference, Kerry and Ledward ${ }^{10}$ used reflectance difference to eliminate the reflectance to attenuance conversion step. However, reflectance intensity did not only depend on the sample, but also varied with different systems. Depending on system sensitivity in a specific wavelength range, reflectance spectra could be different.

\subsection{Statistical Analysis}

A two-tailed, paired sample $t$-test was applied to examine whether the A578-A567 and the A630-A615 differences can be distinguishable between two consecutive days. The A578A567 differences from 104 samples, 52 samples from the first experiment and 52 samples from the second experiment, and the A630-A615 differences from 69 well-bled muscle samples, 34 samples from the first experiment and 35 samples from the second experiment of each two-day pair were used as input for the $t$-test. A $p$-value of less than 0.05 was considered as a statistically significant difference.

\section{Results}

\subsection{Color and Weight Variation Over Time}

Observation of samples showed that from Day0 to Day5 of storage, there was no recognizable change in color. A light brownish-red color was observed on Day6 postmortem and the color change became more visible on Day7. On Day8 and Day9 postmortem, there was juiciness on the surface of the meat. Objective color measurement by quantification of the color change produced a similar outcome (Fig. 5). The significant change of color components occurred from Day6 for the red and green color components and Day 8 for the blue color component. 


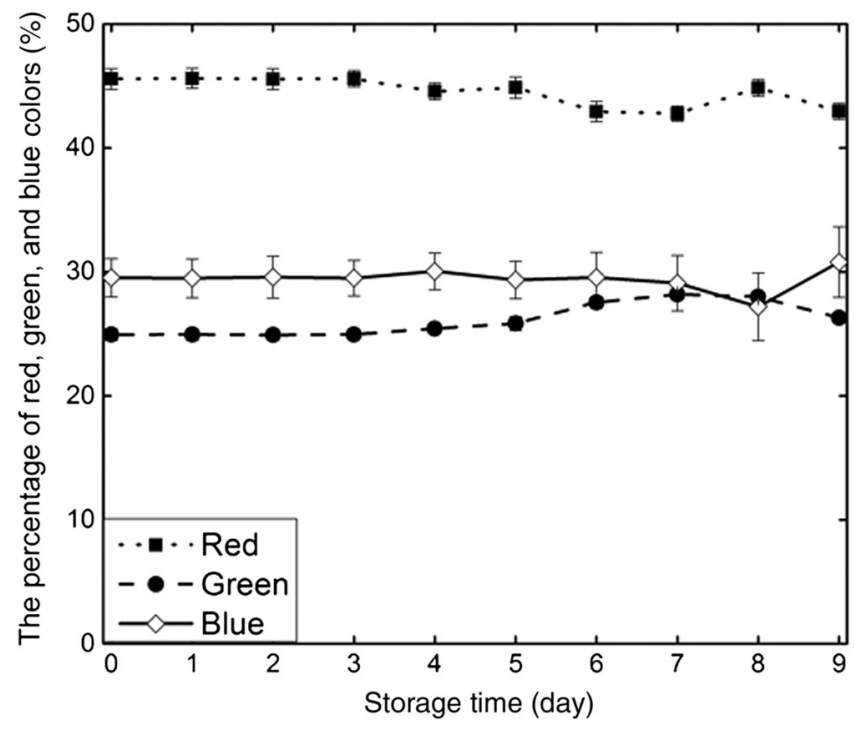

Fig. 5 The averaged percentage of red, green, and blue color components of 69 well-bled muscle samples. (Error bars represent the standard deviation values, but are not shown in green due to their small values.)

The sample weight gradually decreased daily by a small amount. The weight loss after 10 days of storage ranged from $0.2 \%$ in the fat-only sample to $4 \%$ in the well-bled muscle sample. Because fat has little water to lose, it showed a subtle change of weight. Meanwhile, well-bled muscle contains enough water to make its loss significant, hence these samples showed the greatest loss of weight during the storage time. However, weight loss was small enough (up to $4 \%$ ) to assume that the content of myoglobin species remained in the meat, therefore, the reflectance measurements through 10 days have been barely affected by the loss of weight.

\subsection{Attenuation Spectra of Samples}

According to SD and the type of spectrometer, the obtained attenuation spectra of the samples by $\mathrm{S} 1(\mathrm{SD}=3 \mathrm{~mm})$ were from 450 to $1000 \mathrm{~nm}$, and those by $\mathrm{S} 2(\mathrm{SD}=5 \mathrm{~mm}), \mathrm{S} 3$ $(\mathrm{SD}=10 \mathrm{~mm})$, and $\mathrm{S} 4(\mathrm{SD}=15 \mathrm{~mm})$ were from 600 to $1000 \mathrm{~nm}$. Figure 6 shows the attenuance spectra of one wellbled muscle sample collected by S1 from Day0 until Day9; each graph corresponds to a one-day spectrum. Although 19 spectra were obtained from one sample by each spectrometer, for clarity, here only the 10 evening measurement spectra were shown excluding the morning measurements. The attenuance and the shape of the spectrum vary daily. In terms of the attenuance, Day6, Day7, and Day8 show a lower value in the 525 - to $600-\mathrm{nm}$ range, but higher value in the 500- to 525$\mathrm{nm}$ and 600- to 665-nm ranges.

The spectral shape changes greatly with storage time through the variation of the attenuation peaks. Day9 presents only one peak, while the other days express two peaks within the range from 525 to $600 \mathrm{~nm}$. Comparing the two peaks, the second peak position is nearly constant at $578 \mathrm{~nm}$, but the first peak position varies with storage time. On Day0, the first peak is present at $549 \mathrm{~nm}$. After Day0, the peak gradually shifts to a shorter wavelength, and it reaches $544 \mathrm{~nm}$ on Day4. The peak retains its position at $544 \mathrm{~nm}$ until Day6 and then eventually shifts to a higher wavelength. The peak on Day9 is at $550 \mathrm{~nm}$. Together with the peak position shift, the peak amplitude also alters daily, and the most pronounced change is from 557 to $590 \mathrm{~nm}$ and from 610 to $660 \mathrm{~nm}$ (marked by a rectangle in Fig. 6). In the 557- to 590-nm range, a peak appears at $578 \mathrm{~nm}$ from Day0 to Day8 but vanishes on Day9. The peak amplitude rapidly increases from Day0 to Day3, gradually reduces from Day 4 to Day 8, and vanishes on Day9. Additionally, when the peak in the 557- to 590-nm range presents, it has a lower amplitude than the peak in the 542- to 557-nm range. In the 610- to 660-nm range, the attenuance at $630 \mathrm{~nm}$ gradually increases from Day0, resulting in a small peak on Day6. After Day6, the 630-nm attenuance decreases until Day9.

\subsection{Spectral Variation Over Storage Time of Different Sample Types}

Three sample types, well-bled muscle, mixture of muscle and fat, and fat only samples, produce different attenuation spectral variations over storage time. The most pronounced difference occurs in the range from 610 to $660 \mathrm{~nm}$. The 630-nm peak is observed on Day6 in the well-bled muscle and the mixture of muscle and fat samples, but not in the fat only sample. The mixed sample yields a lower attenuance at $630 \mathrm{~nm}$ throughout 10 days' measurement and a smaller $630 \mathrm{~nm}$ attenuance change over storage time compared with the well-bled sample. In the fat sample, there is hardly any change in the 610- to 660$\mathrm{nm}$ spectra, and no peak appearance at $630 \mathrm{~nm}$ during the entire period of measurements.

\subsection{A578-A567 Difference}

Because the A578-A567 difference of the mixture and fat samples produced similar changes as the difference of the well-bled muscle samples, the A578-A567 difference of all three sample types was considered at once. Figure 7 plots the average and the standard deviation of the A578-A567 difference of 104 samples, 52 samples from the first experiment and 52 samples from the second experiment, along with storage time. The averaged A578-A567 difference is positive from Day0 to Day7, and then becomes negative on Day7 to Day9. The difference starts at 0.062 on Day0, increases up to 0.207 on Day4, and decreases down to -0.192 on Day9. The increasing rate is fastest from Day0 to Day1, while the decreasing rate is sharpest from Day6 to Day7. The standard deviation in each measurement day is small compared with the overall relative change.

\subsection{A630-A615 Difference}

Since the spectral change between 610 and $660 \mathrm{~nm}$ varies with sample types over storage time, the averaged difference was calculated separately for each sample type. Figure 8 shows the average and the standard deviation of the A630-A615 difference of 69 well-bled muscle samples from four SDs, 29 mixture samples from the $3 \mathrm{~mm} \mathrm{SD}$, and 6 fat samples from the $3 \mathrm{~mm} \mathrm{SD}$. The A630-A615 difference of well-bled muscle and mixture samples showed a decrease from Day0 to Day6, followed by an increase from Day7 to Day9. In the well-bled muscle samples, the A630-A615 difference is larger with the further SD, and the $10 \mathrm{~mm}$ SD showed the largest change of A630-A615 difference throughout the measurement period. Mixture samples with the $3 \mathrm{~mm}$ SD showed a similar level of A630-A615 difference compared with the well-bled samples, while the fat samples 

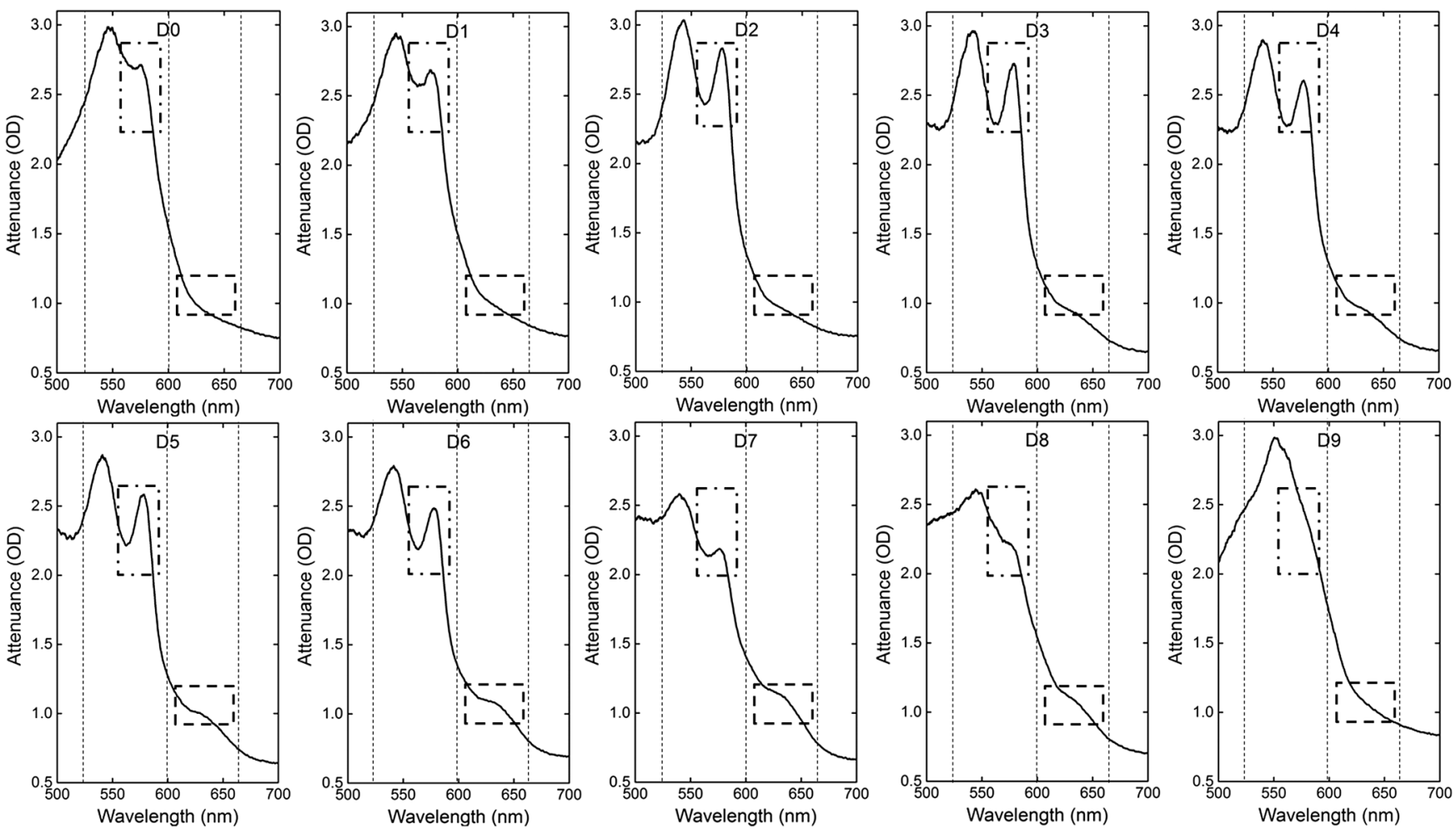

Fig. 6 The 500- to 700-nm attenuation spectra of a well-bled muscle porcine sample.

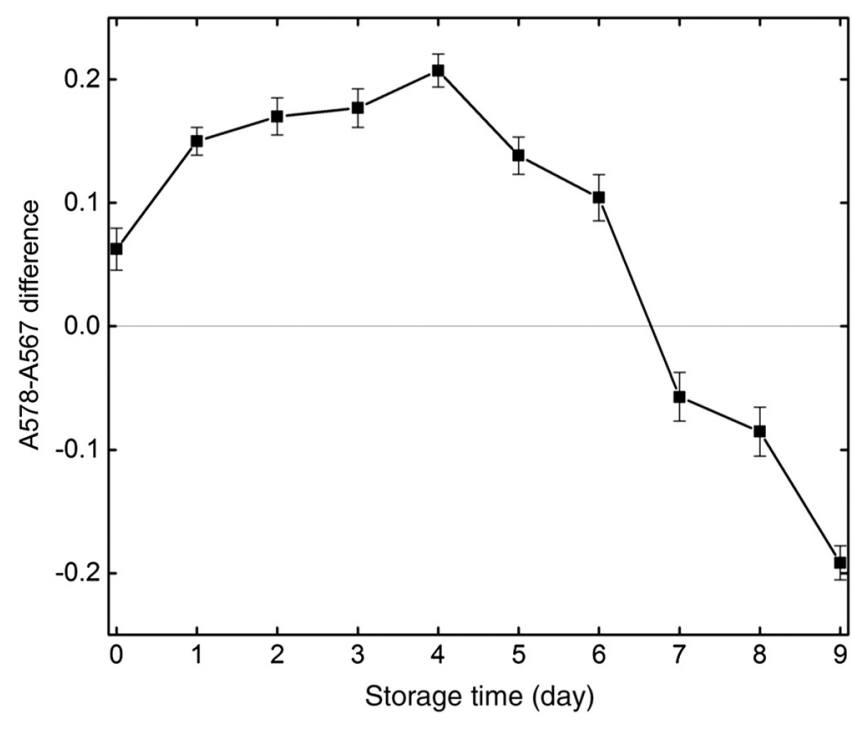

Fig. 7 The average and the standard deviation of the A578-A567 difference of 104 samples (including well-bled muscle, mixture, and fat samples) at $\mathrm{S} 1(\mathrm{SD}=3 \mathrm{~mm})$.

maintained the A630-A615 difference with respect to storage time.

\subsection{Statistical Test}

Table 1 includes the $p$-values of each two-day differences' test. For example, the number in column three, row two is the $p$-value from the $t$-test between A630-A615 differences on Day0 and Day1 of 69 well-bled muscle samples derived from $3 \mathrm{~mm}$ SD. The test yields a small $p$-value for D2-D3 comparison and extremely small $p$-values for all other twoday pairs of the A578-A567 differences. Similarly, most of the two-day pairs of the A630-A615 differences from the four SDs are significantly different, except for D4-D5 in the 10 and $15 \mathrm{~mm}$ SDs and D5-D6 in the 3 and $5 \mathrm{~mm}$ SDs.

\subsection{Oxy-, Deoxy-, and Met-Mb Relative Concentration Changes}

The relative concentration change of the three myoglobin species was calculated from reflectance intensity at 557, 582, and $630 \mathrm{~nm}$ using Beer-Lamberts' law. Figure 9 presents the average and standard variation of oxy-, deoxy-, and met-Mb concentration changes with storage time compared with Day0 of the 69 well-bled and the 29 mixture samples. The relative change has the unit of $\mathrm{mM} / \mathrm{DPF}$, in which DPF is the differential path length factor. ${ }^{28}$ For well-bled muscle and mixture samples, oxy-Mb concentration increases from Day0 to Day4 and decreases after. In opposition, the deoxy-Mb concentration decreases from Day0 to Day4 and increases after. The met-Mb concentration accumulates from Day1 to Day7 and degrades after. In the fat samples, we calculated the $\mathrm{Hb}$ derivatives' concentration, since the fat samples lack muscle but have microblood vessels. Oxy- and deoxy-Hb changes over time have a similar trend as the other two sample types, but the change values are much smaller. The met-Hb change was nearly zero for all days.

\section{Discussion}

From our experiment, we found the gradual increase of the A578-A567 difference from Day0 until Day4 and the decrease of the A630-A615 difference from Day0 until Day6. Following storage time, the relative concentration change increased daily and reached maximum value on Day4 for oxy-Mb and Day 7 for met-Mb. The same trend between the A578-A567 difference 
Table $1 p$-value of the $t$-test between each two-day pair of the A578-A567 differences of 104 samples at $3 \mathrm{~mm} \mathrm{SD}$ and the A630-A615 differences of 69 well-bled samples at $3,5,10$, and $15 \mathrm{~mm}$ SDs.

\begin{tabular}{|c|c|c|c|c|c|}
\hline Day-Day & A578-A567 (3 mm) & A630-A615 (3 mm) & A630-A615 (5 mm) & A630-A615 (10 mm) & $\mathrm{A} 630-\mathrm{A} 615(15 \mathrm{~mm})$ \\
\hline D0-D1 & $<0.001$ & $<0.001$ & $<0.001$ & $<0.001$ & $<0.001$ \\
\hline D1-D2 & $<0.001$ & $<0.001$ & $<0.001$ & $<0.001$ & 0.0068 \\
\hline D2-D3 & 0.0500 & $<0.001$ & 0.0236 & 0.0800 & $<0.001$ \\
\hline D3-D4 & $<0.001$ & 0.0028 & 0.0300 & $<0.001$ & $<0.001$ \\
\hline D4-D5 & $<0.001$ & 0.0224 & $<0.001$ & 0.8696 & 0.1424 \\
\hline D5-D6 & $<0.001$ & 0.8774 & 0.8408 & 0.0136 & 0.035 \\
\hline D6-D7 & $<0.001$ & $<0.001$ & $<0.001$ & 0.0172 & $<0.001$ \\
\hline D7-D8 & $<0.001$ & $<0.001$ & $<0.001$ & $<0.001$ & $<0.001$ \\
\hline D8-D9 & $<0.001$ & $<0.001$ & $<0.001$ & $<0.001$ & $<0.001$ \\
\hline
\end{tabular}

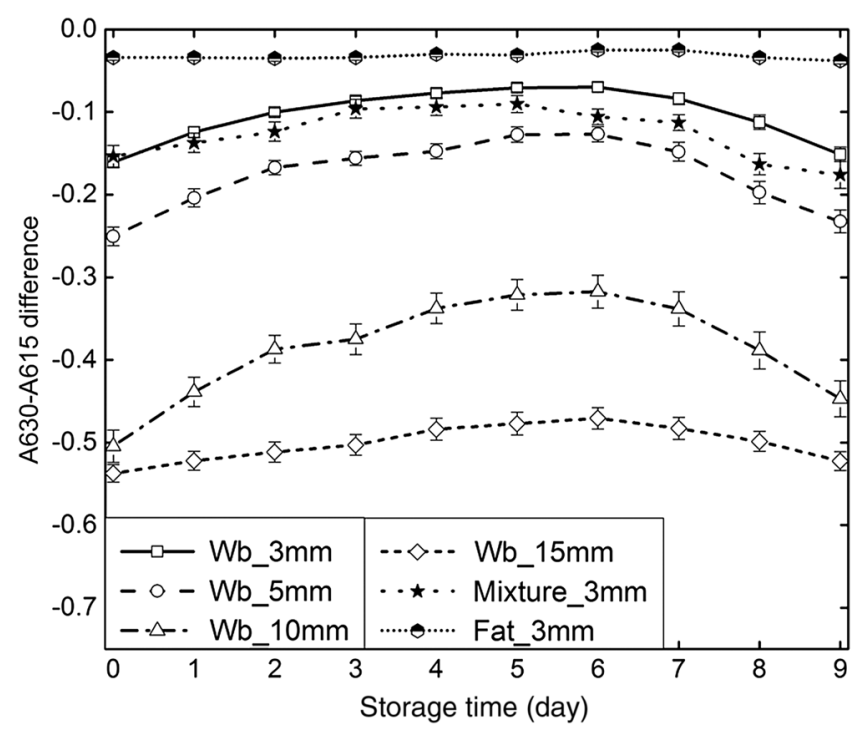

Fig. 8 The A630-A615 difference of 69 well-bled muscle (Wb) (34 from the first and 35 from the second experiment), 29 mixture (16 from the first and 13 from the second experiment), and 6 fat ( 2 from the first and 4 from the second experiment) samples.

(Fig. 7) and oxy- and deoxy-Mb concentration changes (Fig. 9) and between the A630-A615 difference (Fig. 8) and met-Mb concentration change (Fig. 9) during storage time suggested the capability of the attenuance differences to monitor the myoglobin oxygenation and oxidation processes. However, colorimetric analysis did not show a significant change until Day6 for red and green and Day8 for blue. This result proves that the probe with a $3 \mathrm{~mm}$ separation between the source and detector fiber can obtain the myoglobin activities from the inner layer of porcine meat while noncontact colorimetric analysis can show the myoglobin activities only from the surface of porcine meat.

When meat was cut, the cut surface contained mostly deoxy$\mathrm{Mb}$, resulting in the high concentration of this species. After exposure to the air, deoxy-Mb became oxy-Mb, which decreased

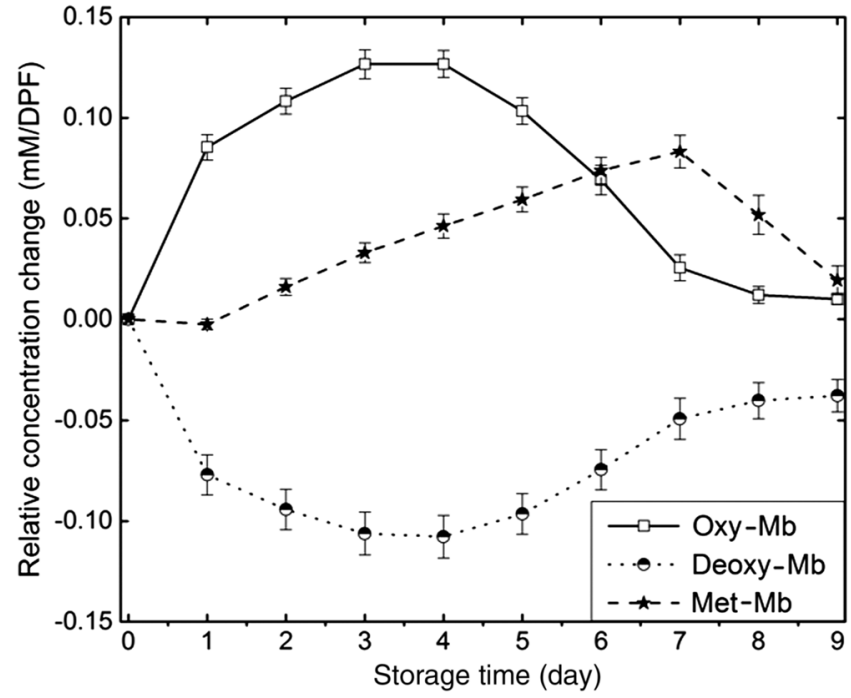

Fig. 9 Oxy-, deoxy-, and met-Mb relative concentration changes of 98 samples (69 well-bled and 29 mixture samples) over storage time.

the deoxy-Mb's portion and increased the oxy-Mb concentration. Following storage time, oxygen was diffused to deeper meat layers and more deoxy-Mb was replaced by oxy-Mb. As a result, during the oxygenation process, oxy-Mb concentration gradually increased while deoxy-Mb concentration partially decreased (Fig. 9); in accordance with the concentration change, the A578-A567 difference increased daily (Fig. 7). Devine and Dikeman $^{7}$ found that the penetration of the oxygen to the meat inner layers continued for several days. In our study, both concentration change (Fig. 9) and the A578-A567 difference (Fig. 7) indicated the termination of the oxygenation process on Day4. The similar tendency of the A578-A567 difference and oxy-Mb after the oxygenation process (from Day5 to Day9) again confirmed the correspondence of the A578-A567 difference to the myoglobin oxygenation process.

Similarly, the matching of the A630-A615 difference (Fig. 8) and met-Mb concentration change (Fig. 9) over storage time 
proved the high correspondence of the A630-A615 difference to met-Mb formation, accumulation, and degradation. On Day0, when the meat samples contained almost no met-Mb, the difference was large (Fig. 8). With time, met-Mb was formed and accumulated (Fig. 9) due to the oxidation process; the difference decreased daily (Fig. 8). After storage for a sufficient time, met$\mathrm{Mb}$ was further oxidized to become free oxidized porphyrins ${ }^{29}$ (met-Mb degradation), which decreased he met-Mb concentration (Fig. 9) and increased the A630-A615 difference. In general, the decrease of the A630-A615 difference signified met-Mb formation and accumulation, while the increase of the difference implied met-Mb degradation.

However, there is a limitation of using the A630-A615 difference to monitor met-Mb activities. After decreasing up to Day6, the difference started to increase from Day7 (Fig. 8), resulting in a mixture of the differences between the days before and after Day6. Therefore, the A630-A615 difference itself cannot tell whether the day of storage was before (met-Mb formation) or after (met-Mb degradation) Day6. Nevertheless, the A578A567 difference becomes negative when the A630-A615 difference starts to increase. Hence, the negative value of the A578A567 difference can be used to distinguish met-Mb degradation from formation and accumulation. Similar to the A630-A615 difference, the A578-A567 difference itself cannot be used to monitor the myoglobin oxygenation process. Though the negative differences on Day7, Day8, and Day9 are unique, the positive differences on other days are mixed due to the decrease of the difference from Day4 to Day6. In order to distinguish the difference from Day0 to Day4 with the difference from Day5 to Day7, we can use the A630-A615 difference, which has a smaller difference from Day5 to Day7 compared with that from Day0 to Day4.

In general, from our experiments, we found that instead of investigating the whole frequency spectrum of the meat sample, it is possible to use multiwavelength measurement to monitor myoglobin biological activities. Specifically, the relative concentration change of three myoglobin species can be calculated from the reflectance intensities at 557, 582, and $630 \mathrm{~nm}$ and the oxygenation and oxidation processes can be investigated from the A578-A567 and A630-A615 differences. Instead of using a broadband light source with a spectrometer, our study's result suggests a new method, which consists of LEDs and a photodetector, to monitor myoglobin biological activities. The low cost of the LED and photodetector enables our technique to be widely applied in real life. In addition, our method was able to detect the formation of met-Mb much earlier than the colorimetric analysis.

\section{Conclusions}

In summary, an approach to monitor myoglobin oxygenation and met-Mb formation, accumulation, and degradation in porcine meat was proposed. The experimental results show the potential of the early detection of met-Mb formation, before the onset of visible color change. Furthermore, the combination of the A630-A615 difference and the A578-A567 difference can provide information about myoglobin oxygenation and met-Mb formation and degradation, suggesting a practical alternative to using a wide range of spectra from a spectrometer. In addition, instead of collecting the whole spectrum, measurement of four wavelengths' attenuance $(567,578,615$, and $630 \mathrm{~nm})$ simplifies the system hardware and speeds up computation of the differences. Based on our results, we believe that this simple multiwavelength attenuance measurement can be a promising method for monitoring myoglobin oxygenation and also the formation, accumulation, and degradation of met-Mb.

\section{Acknowledgments}

This work was partially supported by the National Research Foundation of Korea (NRF) Grants (2012K1A2B1A03000757, 2013R1A1A2013625, and 2015R1D1A1A02062382), a grant (CRI12071-3) of the CNUH-GIST, the Traditional Korean Medicine R\&D Program funded by the Ministry of Health and Welfare through the Korea Health Industry Development Institute (HI15C0190), and a grant from the Small and Medium Business Administration (S2273981).

\section{References}

1. M. Du and R. J. McCormick, Eds., Applied Muscle Biology and Meat Science, CRC Press, Boca Raton, Florida (2009).

2. R. A. Mancini and M. Hunt, "Current research in meat color," Meat Sci. 71(1), 100-121 (2005).

3. Y. H. Hui, W. K. Nip, and R. Rogers, Eds., Meat Science and Applications, CRC Press, Boca Raton, Florida (2001).

4. S. J. Millar, B. W. Moss, and M. H. Stevenson, "Some observations on the absorption spectra of various myoglobin derivatives found in meat," Meat Sci. 42(3), 277-288 (1996).

5. Y. H. Hui, Ed., Handbook of Meat and Meat Processing, CRC Press, Boca Raton, Florida (2012).

6. D. J. Livingston and W. D. Brown, "The chemistry of myoglobin and its reactions [meat pigments, food quality indices]," Food Technol. 35(5), 238-252 (1981)

7. C. Devine and M. Dikeman, Eds., Encyclopedia of Meat Sciences: 3-Volume Set, Elsevier, Philadelphia, Pennsylvania (2014).

8. J. A. Boles and R. Pegg, "Meat color," in Montana State University and Saskatchewan Food Product Innovation Program, University of Saskatchewan, Saskatoon, Saskatchewan (2010).

9. P. D. Warriss, Meat Science: An Introductory Text, 2nd ed., pp. 113129, Cabi, Wallingford, Oxfordshire, United Kingdom (2010).

10. J. P. Kerry and D. Ledward, Eds., Improving the Sensory and Nutritional Quality of Fresh Meat, Elsevier, Philadelphia, Pennsylvania (2009).

11. S. Homma, T. Fukunaga, and A. Kagaya, "Influence of adipose tissue thickness on near infrared spectroscopic signal in the measurement of human muscle," J. Biomed. Opt. 1(4), 418-424 (1996).

12. W. J. Bowen, "The absorption spectra and extinction coefficients of myoglobin,” J. Biol. Chem. 179(1), 235-245 (1949).

13. Y. Liu and Y. R. Chen, "Analysis of visible reflectance spectra of stored, cooked and diseased chicken meats," Meat Sci. 58(4), 395-401 (2001).

14. C. N. Pace et al., "How to measure and predict the molar absorption coefficient of a protein," Protein Sci. 4(11), 2411-2423 (1995).

15. K. A. Schenkman et al., "Myoglobin oxygen dissociation by multiwavelength spectroscopy," J. Appl. Physiol. 82(1), 86-92 (1997).

16. K. Krzywicki, "Assessment of relative content of myoglobin, oxymyoglobin and metmyoglobin at the surface of beef," Meat Sci. 3(1), 1-10 (1979).

17. J. P. Kerry, J. F. Kerry, and D. Ledward, Eds., Meat Processing: Improving Quality, Elsevier, Philadelphia, Pennsylvania (2002).

18. S. P. Nighswander-Rempel, V. V. Kupriyanov, and R. A. Shaw, "Relative contributions of hemoglobin and myoglobin to near-infrared spectroscopic images of cardiac tissue," Appl. Spectrosc. 59(2), 190193 (2005).

19. C. Sen, L. Packer, and O. Hänninen, Eds., Handbook of Oxidants and Antioxidants in Exercise, Elsevier, Philadelphia, Pennsylvania (2000).

20. W. G. Zijlstra, A. Buursma, and W. P. Meeuwsen-Van der Roest, "Absorption spectra of human fetal and adult oxyhemoglobin, de-oxyhemoglobin, carboxyhemoglobin, and methemoglobin," Clin. Chem. 37(9), 1633-1638 (1991).

21. S. L. Jacques, "Optical properties of biological tissues: a review," Phys. Med. Biol. 58(11), R37 (2013). 
22. R. N. Arnold et al., "Visual and spectrophotometric evaluations of beef color stability," J. Food Sci. 57(2), 518-520 (1992).

23. W. C. Franke and M. Solberg, "Quantitative determination of metmyoglobin and total pigment in an intact meat sample using reflectance spectrophotometry," J. Food Sci. 36(3), 515-519 (1971).

24. L. M. Nollet, Ed., Handbook of Meat, Poultry and Seafood Quality, John Wiley \& Sons, Hoboken, New Jersey (2012).

25. R. A. Mancini, M. C. Hunt, and D. H. Kropf, "Reflectance at 610 nanometers estimates oxymyoglobin content on the surface of ground beef," Meat Sci. 64(2), 157-162 (2003).

26. M. C. Hunt et al., "Guidelines for meat color evaluation," in 44th Annual Reciprocal Meat Conf., American Meat Science Association, Savoy, Illinois (1991).

27. A. N. Bashkatov, E. A. Genina, and V. V. Tuchin, "Optical properties of skin, subcutaneous, and muscle tissues: a review," J. Innovative Opt. Health Sci. 4(01), 9-38 (2011).

28. E. Kim et al., "A discrepancy of penile hemodynamics during visual sexual stimulation observed by near-infrared spectroscopy," $B M C$ Urol. 15(1), 11 (2015).

29. N. N. Potter and J. H. Hotchkiss, Food Science, Springer Science \& Business Media, New York (2012).

Thien Nguyen received her BS degree in biomedical engineering from the Hanoi University of Science and Technology, Hanoi, Vietnam, in 2013 and her MS degree in medical system engineering from the Gwangju Institute of Science and Technology, Gwangju, Korea, in 2015. She is a PhD candidate in the Biophotonics Lab, Department of Biomedical Science and Engineering, Gwangju
Institute of Science and Technology, Gwangju, Korea. Her current research interest is in translational optics for medical applications.

Kien Nguyen Phan is a lecturer in Hanoi University of Science and Technology and a member of Japan Society of Physiology. He received his BS and MS degrees from the School of Electronics and Telecommunication in the Hanoi University of Science and Technology, Vietnam, in 1999 and 2002, respectively. He graduated with his PhD degree from Shibaura Institute of Technology, Japan, in 2005. His research interest is in biomechanics, meat science and applied electronics for medical application.

Jee-Bum Lee received his MD degree from Chonnam National University Medical School, Gwangju, Republic of Korea, in 1989 and his MS and PhD degrees in medical science from Graduate School of Chonnam National University, Gwangju, in 1997 and 2000 , respectively. He is currently the professor and chairman of Department of Dermatology, Chonnam National University Medical School.

Jae Gwan Kim is a professor in the Department of Biomedical Science and Engineering, Gwangju institute of Science and Technology (GIST), Gwangju, Republic of Korea. Before joining GIST, he was a postdoctoral scholar at the Beckman Laser Institute and Medical Clinic, University of California, Irvine. He received his PhD from Joint Program of Biomedical Engineering between the University of Texas Arlington and the UT Southwestern Medical Center at Dallas in 2005. 